\title{
Network pharmacology modeling identifies synergistic interaction of therapeutic and toxicological mechanisms for Tripterygium hypoglaucum Hutch
}

\author{
Dan Zhang ${ }^{1}$, Yizhu Dong ${ }^{1}$, Jintao Lv', Bing Zhang ${ }^{1,2^{*}} \mathbb{D}^{1}$, Xiaomeng Zhang ${ }^{1}$ and Zhijian Lin $^{1}$
}

\begin{abstract}
Background: Tripterygium hypoglaucum Hutch (THH) both has prominent efficacy and unwarranted toxicity in the treatment of autoimmune diseases. Nevertheless, its pharmacological and toxicological profiles still remain to be elucidated. In the current study, the network pharmacology approach was applied to identify synergistic interaction and mechanism of efficacy and toxicity for THH from a holistic perspective.

Methods: The compounds from THH were collected using literature retrieval and relevant databases. After the production of putative therapeutic targets for dominant diseases and harmful targets of adverse reactions (ADRs) induced by $\mathrm{THH}$, the protein-protein interactions (PPIs), topological analysis and pathway enrichment were established to distinguish the hub targets and pathways. Additionally, the binding activity of candidate ingredients with core targets were revealed by molecular docking simulation.

Results: A total of eight bioactive components in THH were enrolled, and 633 targets were responsible for rheumatoid arthritis (RA), 1067 targets were corresponding to systemic lupus erythematosus (SLE), 1318 targets of ADRs were obtained. The results of enrichment analysis among THH-RA, THH-SLE and THH-ADR networks indicated that pathway in cancer, hepatitis B, rheumatoid arthritis, and PI3K-Akt signaling pathway might participate in THH for treating RA and SLE. Besides, the mechanism of ADRs that induced by THH were associated with viral carcinogenesis, p53 signaling pathway, PI3K-Akt signaling pathway, and so on. Whereas, these active ingredients of THH exerted the superior binding activities with crucial targets including STAT3, VEGFA, TP53 and MMP9 that functioned synergistically efficacy and toxicity as observed via molecular docking simulation.

Conclusion: The present research preliminarily interpreted the synergistic interaction of therapeutic and toxicological mechanisms for THH through the comprehensive analysis of relationship and binding activity between primary components and core targets, providing a feasible and promising approach to facilitate the development of toxic and irreplaceable herbs.
\end{abstract}

Keywords: Network pharmacology, Molecular docking, Tripterygium hypoglaucum Hutch, Mechanisms, Efficacy, Toxicity

\footnotetext{
* Correspondence: zhangb@bucm.edu.cn

'Department of Clinical Chinese Pharmacy, School of Chinese Materia

Medica, Beijing University of Chinese Medicine, No. 11 North Three-ring East

Road, Chao Yang District, Beijing 100102, China

${ }^{2}$ Center for Pharmacovigilance and Rational Use of Chinese Medicine, Beijing

University of Chinese Medicine, Beijing 102488, China
}

(C) The Author(s). 2021 Open Access This article is licensed under a Creative Commons Attribution 4.0 International License, which permits use, sharing, adaptation, distribution and reproduction in any medium or format, as long as you give appropriate credit to the original author(s) and the source, provide a link to the Creative Commons licence, and indicate if changes were made. The images or other third party material in this article are included in the article's Creative Commons licence, unless indicated otherwise in a credit line to the material. If material is not included in the article's Creative Commons licence and your intended use is not permitted by statutory regulation or exceeds the permitted use, you will need to obtain permission directly from the copyright holder. To view a copy of this licence, visit http://creativecommons.org/licenses/by/4.0/. The Creative Commons Public Domain Dedication waiver (http://creativecommons.org/publicdomain/zero/1.0/) applies to the data made available in this article, unless otherwise stated in a credit line to the data. 


\section{Background}

As a typical Chinese herb with promising immunosuppressive and anti-inflammatory bioactivities, Tripterygium hypoglaucum (level) Hutch (THH), named as KunmingShanhaitang in Mandarin, is a traditional Chinese materia medica belonging to the family Celastraceae and the genus Tripterygium [1, 2]. According to the theory of traditional Chinese medicine (TCM), the medicinal effects of THH including dispelling wind and removing dampness, eliminating blood stasis and dredging collaterals, have been first dissertated in Compendium of Materia Medica, and it is warm in nature, acrid and bitter in flavor, with toxicity $[3,4]$. Interestingly, with respect to morphologically similar species from Tripterygium genus, THH exhibits the similarity on therapeutic effects, clinical indications and toxicity with Tripterygium wilfordii Hook, besides, it has been widely affirmed in treating chronic autoimmune diseases due to mild pharmaceutical advantages and lasting immunosuppressive effects [5]. Currently, THH and its tablet preparations have played a desirable and beneficial role in the treatment of rheumatoid arthritis (RA), systemic lupus erythematous (SLE), and other autoimmune or inflammatory diseases for decades $[6,7]$. The bioactive principles of $\mathrm{THH}$, namely sesquiterpenoids, diterpenes, triterpenoids and so on, exert valuable and excellent pharmacological profiles of anti-inflammation, analgesia and immunomodulatory activities [8-10].

Although THH achieves considerably curative efficacy, with an overdose or prolonged exposure in clinic and its narrow therapeutic window, the application of THH has been impeded due to undesirable side effects and multiple organ toxicities including hepatotoxicity, nephrotoxicity, reproductive damage, and cardiotoxicity [11-13]. Accumulating evidence suggests that $\mathrm{THH}$ and its active ingredients could induce severe adverse reactions (ADRs), particularly hepatotoxicity and nephrotoxicity, these side effects can not only display the higher serum levels of alanine transaminase, aspartate transaminase, serum creatinine and urea nitrogen, but also are responsible for inhibiting CYP450 and the transporters [14-17]. Moreover, the clinical manifestation of reproductive system function impairments induced by correlative components of THH involving premature ovarian insufficiency, menstrual cycle irregularities, oligospermia and so forth [18-21]. Unfortunately, the gastrointestinal reactions including nausea, vomitus, abdominalgia and diarrhea, cardiovascular toxicities covering chest distress and palpitation had contributed to the limitation of its immense therapeutic potentials [22-24]. Remarkably, the irreplaceable merits of THH against rheumatic diseases are accompanied by side effects, therefore, its safety issues have raised major concerns. Nevertheless, current toxicological studies often focus on acute toxicity, reproductive toxicity and nephrotoxicity for its similar species from Tripterygium genus, Tripterygium wilfordii Hook, or some components from $\mathrm{THH}$
[25-29]. With this in mind, the approaches of network pharmacology combined with molecular docking were employed to distinguish bioactive components and predict key targets, for disclosing synergistic interaction of efficacious and toxic mechanisms for THH from a systemic and holistic perspective.

\section{Methods}

The current study was proposed by four-step analysis including data preparation, network construction, enrichment analysis and molecular docking simulation, the flowchart of technical strategy in present study is illustrated in Fig. 1.

\section{Data preparation}

\section{Candidate components screening}

As the world's largest non-commercial TCM database available for drug screening in silico, herbal compounds of THH were collected and extracted from TCM Database@Taiwan (http://tcm.cmu.edu.tw) [30]. In addition, the potentially eligible compounds were recruited through the electronic databases of PubMed, the China National Knowledge Infrastructure Database, the Wanfang Database, and the China Biology Medicine disc. Then, these components groups of THH were selected according to Lipinski's Rule of Five, Veber's rule and Muegge's rule as potential therapeutic candidates [31, 32]. Moreover, the chemical information including MOL2 structure, canonical name and SMILES number were employed and downloaded for subsequent computational analysis from the PubChem Compound Database (http:// pubchem.ncbi.nlm.nih.gov/) [33] and Traditional Chinese Medicine Systems Pharmacology Database (TCMSP, http://ibts.hkbu.edu.hk/LSP/tcmsp.php) [34].

\section{Putative targets of $\mathrm{THH}$}

The targets data of main compounds from THH were identified and implemented from following databases: 1) Corresponding targets of all the promising components were captured from TCMSP Database. 2) The SMILES number of each candidate ingredient was imported into SWISS (http://www.swisstargetprediction.ch/) to acquire targets data of THH. This web server was designed to accurately identify potential targets of bioactive molecules according to a combination of $2 \mathrm{D}$ and $3 \mathrm{D}$ similarity measures with known ligands [35]. Targets with probability score $\geq 0.7$ were selected for further analysis. 3) The predicted target profiles were also obtained from STITCH (Version 5.0, http://stitch.embl.de/) according to SMILES number of each molecule [36]. After discarding duplicates, the results of all targets prediction from above approaches were standardized into target genes names by utilizing Uniport sites (http://www.uniprot. org/) [37, 38], ultimately, these target genes from Homo 


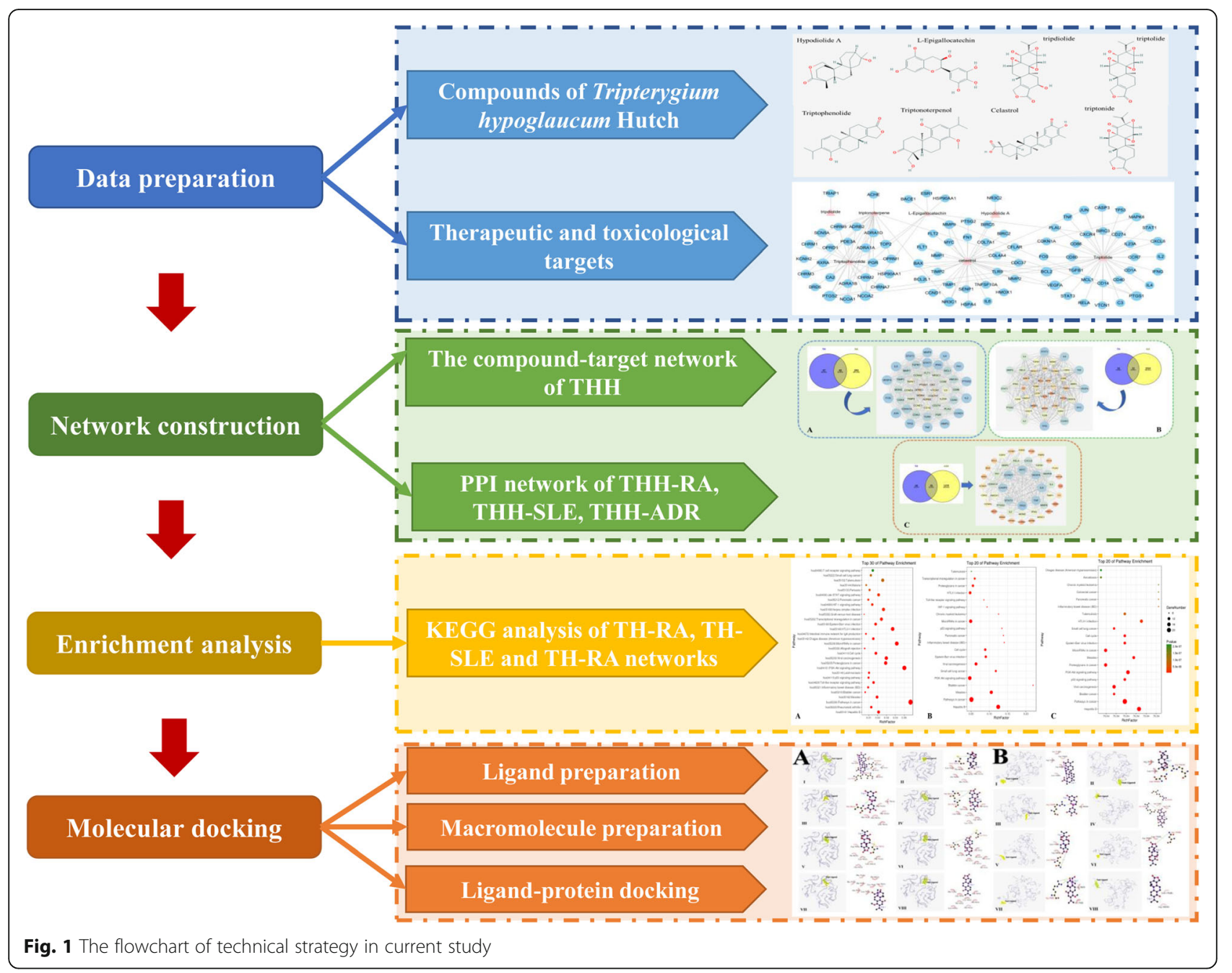

sapiens were harvested altogether as targets database of $\mathrm{THH}$.

\section{Related targets of RA and SLE}

According to Chinese Pharmacopoeia (2015 Edition) [39], the dominant diseases of THH were RA and SLE. Therefore, the keywords including "rheumatoid arthritis, rheumatoid, arthritis, rheumatoid nodule, rheumatoid arthritis" and "systemic lupus erythematosus, lupus erythematosus disseminates, Libman-Sacks disease, Libman Sacks disease, verrucous endocarditis" as the queries were searched for known therapeutic targets from the Online Mendelian Inheritance in Man (OMIM, http:// omim.org/) [40], the Therapeutic Target Database (TTD, http://bidd.nus.edu.sg/group/cittd/) [41] and Genetic Association Database (GAD, https://geneticassociationdb. nih.gov/) [42].

\section{Associated targets of ADRs}

Increasing experimental evidence has verified that $\mathrm{THH}$ could induce multiple organ toxicities including nephrotoxicity, hepatotoxicity, reproductive toxicity, cardiotoxicity and other side effects. The keywords of ADRs were searched in Comparative Toxicogenomics Database (CTD, http://ctdbase.org) to obtain the toxicological targets. Notably, CTD was a robust, publicly available research resource with over 30.5 million toxicogenomic relationships [43]. In addition, the searching entries of ADRs involved nephrotoxicity ("acute renal injury"), hepatotoxicity ("acute/chronic/drug-induced liver injury"), reproductive toxicity ("amenorrhea, menstrual disorder"), cardiac toxicity ("arrhythmia, palpitation"), hematological toxicity ("anemia, leukopenia"), gastrointestinal toxicity ("nausea, vomiting"), skin lesions ("rash, pruritus") and so on.

\section{Network construction}

First, the putative targets of active ingredients from THH were mapped to different targets' group of dominant diseases and ADRs, which were regarded as potential efficacy and toxicity targets, respectively. Second, these candidate targets were inputted into the Search Tool for the Retrieval of Interacting Genes/Proteins (STRING, 
https://string-db.org/) to forecast three protein-protein interactions (PPIs), namely THH against RA (THH-RA), THH against SLE (THH-SLE) and THH caused ADR (THH-ADR), with the species limited to "Homo sapiens" and the confidence scores were higher than 0.4 [44]. Subsequently, the visualization and analysis of PPIs were undertaken through Cytoscape software [45]. Finally, the intersections of three PPIs including THH-RA, THHSLE and THH-ADR were generated to identify core targets which were responsible for both clinical benefits and harms, and each hub target in the area of intersection was assessed and estimated with its typical central attributes: betweenness centrality (BC), closeness centrality $(C C)$, and degree centrality (DC).

\section{Enrichment analysis}

The Database for Annotation, Visualization, and Integrated Discovery Bioinformatics Resources (DAVID, https:// david.ncifcrf.gov/, version 6.8) [46], a web-based online bioinformatics resource for the high-throughput functional annotation bioinformatics functional annotation and enrichment analysis, was conducted to perform for Kyoto Encyclopedia of Genes and Genomes (KEGG, http://www. kegg.jp/) pathway enrichment, with $p$ value $<0.05$ was considered statistically significant [47]. The visualization of enrichment analysis was illuminated through Omicshare cloud platform (http://www.omicshare.com/).

\section{Molecular docking simulation}

Molecular docking simulation included three different steps, namely ligand preparation, macromolecule preparation and ligand-protein docking. The interactions and binding models between the candidate components and the crucial pharmacodynamic and toxic protein were verified by molecular docking via the Systems Dock Web Site (http://systemsdock.unit.oist.jp/) [48], which permitted molecular docking simulation for comprehensive characterization of ligand selectivity and action on a complex molecular network. First, the 3D molecular structure of each compound was captured from PubChem website and then imported to AutoDock Tools 1.5.6 (The Scripps Research Institute, Santiago, California and the United States) for removing water molecules, adding hydrogenate, and charging energy; the files of optimized small molecules were saved as mol2 format ultimately [49]. Second, the crystal structures and protein conformation of core targets were obtained and downloaded from Protein Data Bank (PDB) database (http://www.rcsb.org/pdb/home/ home.do) [50]. Third, the definition of binding sites was established through an interactive molecular visualizer by clicking on the displayed structure model or residues listed. In addition, the docking scores which were reported by docK-IN ranged from 0 to 10 (from weak to strong binding), allowing a straightforward indication of binding strength. Briefly, the compounds with higher docking scores more than $7.0 \mathrm{pKd} / \mathrm{pKi}$ indicated that it possessed strong binding affinity with target receptors, and docking scores more than $5.0 \mathrm{pKd} / \mathrm{pKi}$ equaled moderate binding affinity [48].

\section{Results}

Identification of components and targets

A total of 36 chemical ingredients of THH were obtained from the literature and TCM Database@Taiwan, ultimately, eight of them were regarded as bioactive ingredients by filtering with Lipinski's Rule of Five, Veber's rule and Muegge's rule. The chemical information (chemical name, chemical formula, molecular weight and so on) and structure of active ingredients are displayed in Table 1 and Fig. 2, respectively. Altogether, 87 potential targets were acquired from previously described after removing the redundancy, and the compound-target network is depicted in Fig. 3.

\section{Network construction of dominant diseases and ADRs}

The target prediction of THH in treating RA (targets number $=633$ ) and SLE (targets number $=1067$ ) and inducing ADRs (targets number $=1318$ ) were achieved through online databases and relevant software. As illustrated in Fig. 4, the intersection of these putative therapeutic and toxicological targets of THH was generated

Table 1 The chemical information of active ingredients from $\mathrm{THH}$

\begin{tabular}{lllllllllll}
\hline Chemical name & $\begin{array}{l}\text { Chemical } \\
\text { formula }\end{array}$ & $\begin{array}{l}\text { Molecular } \\
\text { weight }\end{array}$ & $\begin{array}{l}\text { ALogP } \\
\end{array}$ & $\begin{array}{l}\text { Num. } \\
\text { H-bond } \\
\text { acceptors }\end{array}$ & $\begin{array}{l}\text { Num. } \\
\text { H-bond } \\
\text { donors }\end{array}$ & $\begin{array}{l}\text { Num. } \\
\text { rotatable } \\
\text { bonds }\end{array}$ & $\begin{array}{l}\text { Consensus } \\
\text { Log Po/w }\end{array}$ & $\begin{array}{l}\text { Log S } \\
\text { (ESOL) }\end{array}$ & $\begin{array}{l}\text { Log Kp } \\
\text { (cm/s) }\end{array}$ & $\begin{array}{l}\text { Bioavailability } \\
\text { Score }\end{array}$ \\
\hline Hypodiolide A (Tripterifordin) & $\mathrm{C}_{20} \mathrm{H}_{30} \mathrm{O}_{3}$ & 318.45 & 3.248 & 3 & 1 & 0 & 3.63 & -4.23 & -5.52 & 0.55 \\
Hypolide (Triptophenolide) & $\mathrm{C}_{20} \mathrm{H}_{24} \mathrm{O}_{3}$ & 312.4028 & 4.63 & 2 & 1 & 1 & 3.91 & -4.46 & -5.33 & 0.55 \\
L-Epigallocatechin & $\mathrm{C}_{15} \mathrm{H}_{14} \mathrm{O}_{7}$ & 306.267 & 1.779 & 0 & 0 & 1 & 0.42 & -2.08 & -8.17 & 0.55 \\
Tripdiolide & $\mathrm{C}_{20} \mathrm{H}_{24} \mathrm{O}_{7}$ & 376.4 & -0.23 & 0 & 0 & 1 & 0.90 & -1.55 & -9.23 & 0.55 \\
Triptolide & $\mathrm{C}_{20} \mathrm{H}_{24} \mathrm{O}_{6}$ & 360.401 & 0.872 & 0 & 0 & 1 & 1.71 & -2.15 & -8.34 & 0.55 \\
Triptonide & $\mathrm{C}_{20} \mathrm{H}_{22} \mathrm{O}_{6}$ & 358.385 & 1.259 & 0 & 0 & 1 & 1.93 & -2.41 & -8.02 & 0.55 \\
Triptonoterpenol & $\mathrm{C}_{21} \mathrm{H}_{30} \mathrm{O}_{4}$ & 346.461 & 3.895 & 4 & 2 & 3 & 3.63 & -4.59 & -5.45 & 0.55 \\
Celastrol (tripterine) & $\mathrm{C}_{29} \mathrm{H}_{38} \mathrm{O}_{4}$ & 450.619 & 5.9 & 4 & 2 & 1 & 5.12 & -6.31 & -4.83 & 0.85 \\
\hline
\end{tabular}




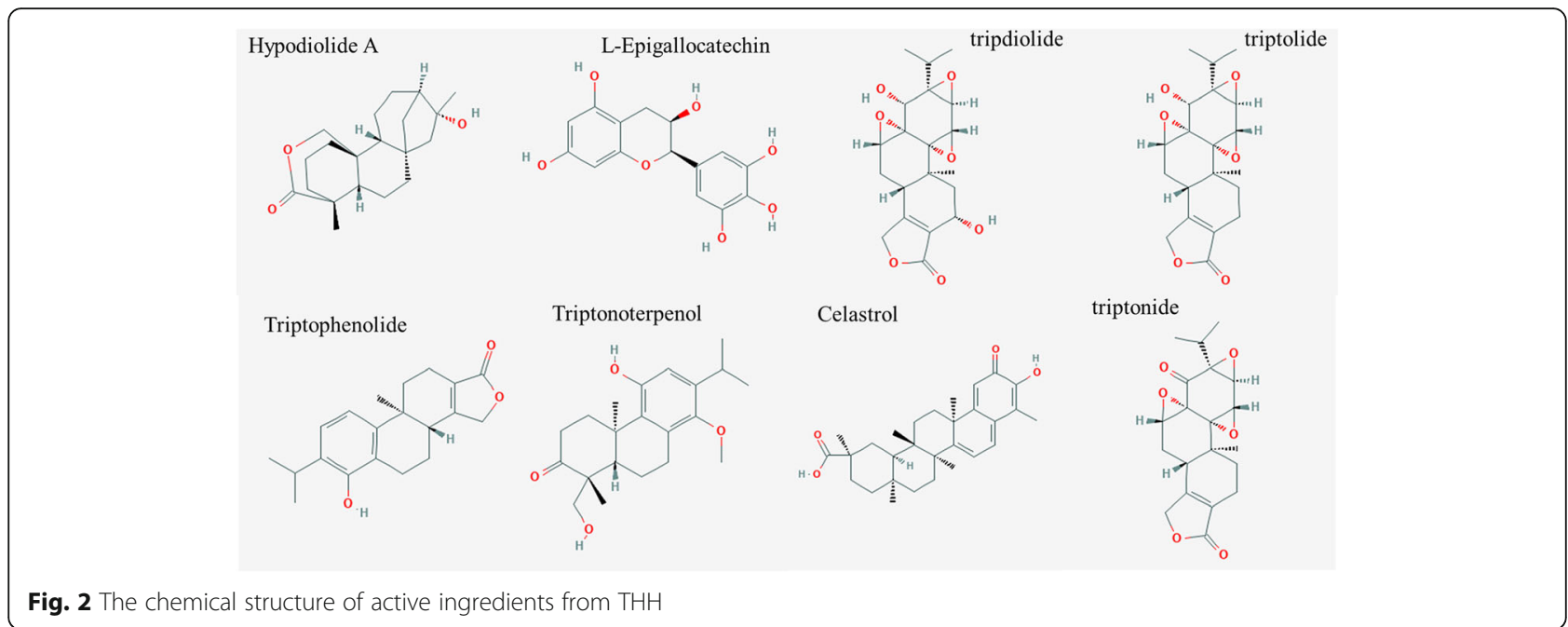

and imported into STRING database to establish three PPIs including THH-RA, THH-SLE and THH-ADR. Based on these PPIs network, RA-SLE-ADR network was constructed for understanding the concurrent mechanism of efficacy and toxicity for THH. According to statistical analysis results of topological parameters, significant targets with the higher degree is summarized in Fig. 5 and Table 2. From yellow to blue, the degree of targets was increasing, and the thicker edges represented the stronger interactions, thereby indicating that the top mutual targets of THH had both beneficial and harmful functions at the molecular level.

\section{KEGG enrichment analysis}

The pathways enrichment analysis of candidate targets among THH-RA, THH-SLE and THH-ADR networks was performed by utilizing DAVID platform. The corresponding results elucidated that pathway in cancer, hepatitis B, rheumatoid arthritis, PI3K-Akt signaling pathway and measles were represented as pathways of THH for treating RA and SLE. Additionally, the ADRs caused by THH were associated with viral carcinogenesis, p53 signaling pathway, PI3K-Akt signaling pathway, pathways in cancer, and bladder cancer (Fig. 6).

\section{Molecular docking simulation}

Among RA-SLE-ADR network, the key targets with higher degree including IL6 (Interleukin-6 receptor), STAT3 (Signal transducers and activators of transcription30, TNF (Tumor necrosis factor), VEGFA (Vascular endothelial growth factor), TP53 (Cellular tumor antigen p53), MMP9 (Matrix metalloprotein 9).

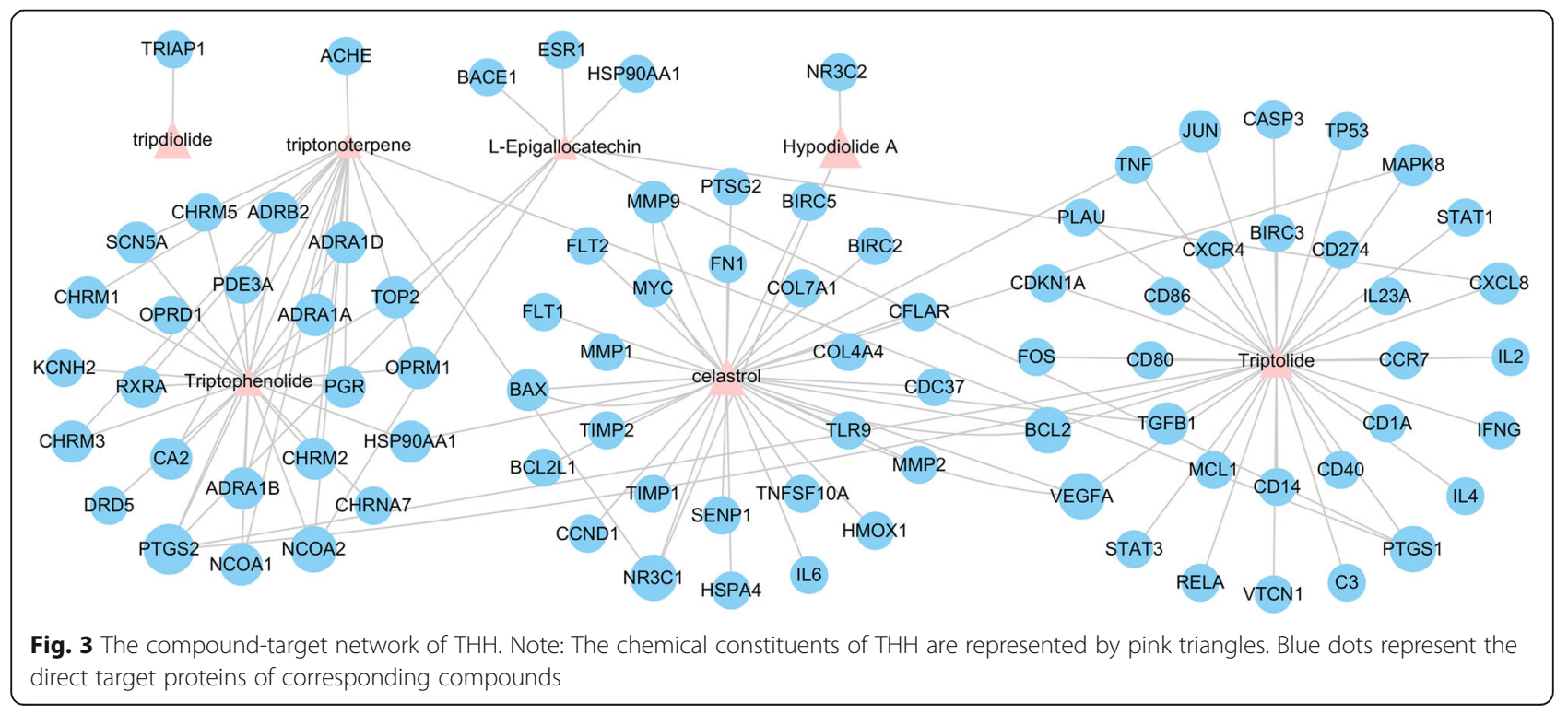




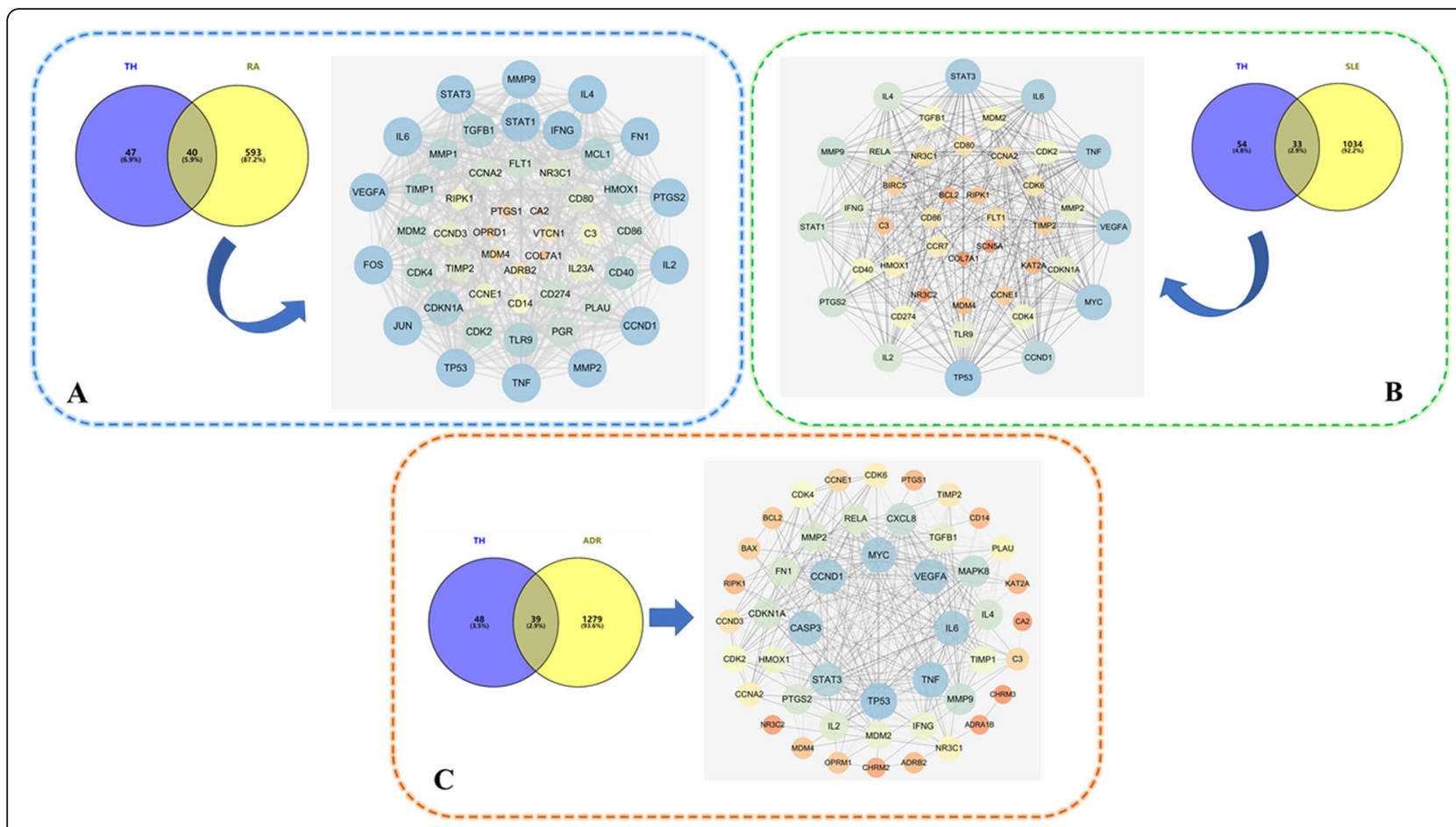

Fig. 4 The PPI networks associating with the efficacy and toxicity of THH. Note: a: The PPI network of THH for treating rheumatoid arthritis (RA). b: The PPI network of THH for treating systemic lupus erythematosus (SLE). c: The PPI networks of THH induced adverse reactions (ADRs)

Remarkably, in consideration of IL6 and TNF were inflammatory factors with the inferior specificity in current research. Consequently, STAT3, VEGFA, TP53 and MMP9 were identified as corresponding receptors that possessed both efficacy and toxicity simultaneously for further process of molecular docking simulation. The four targets were searched in PDB database, and their configurations were determined as 1BG1, 3DCY, 4DEQ and 1ITV.

To provide the deeper insight into the binding interactions between active principles of $\mathrm{THH}$ and these core targets, collectively, the results showed that each ingredient yielded moderate binding activity, suggesting there were the potentials of direct binding
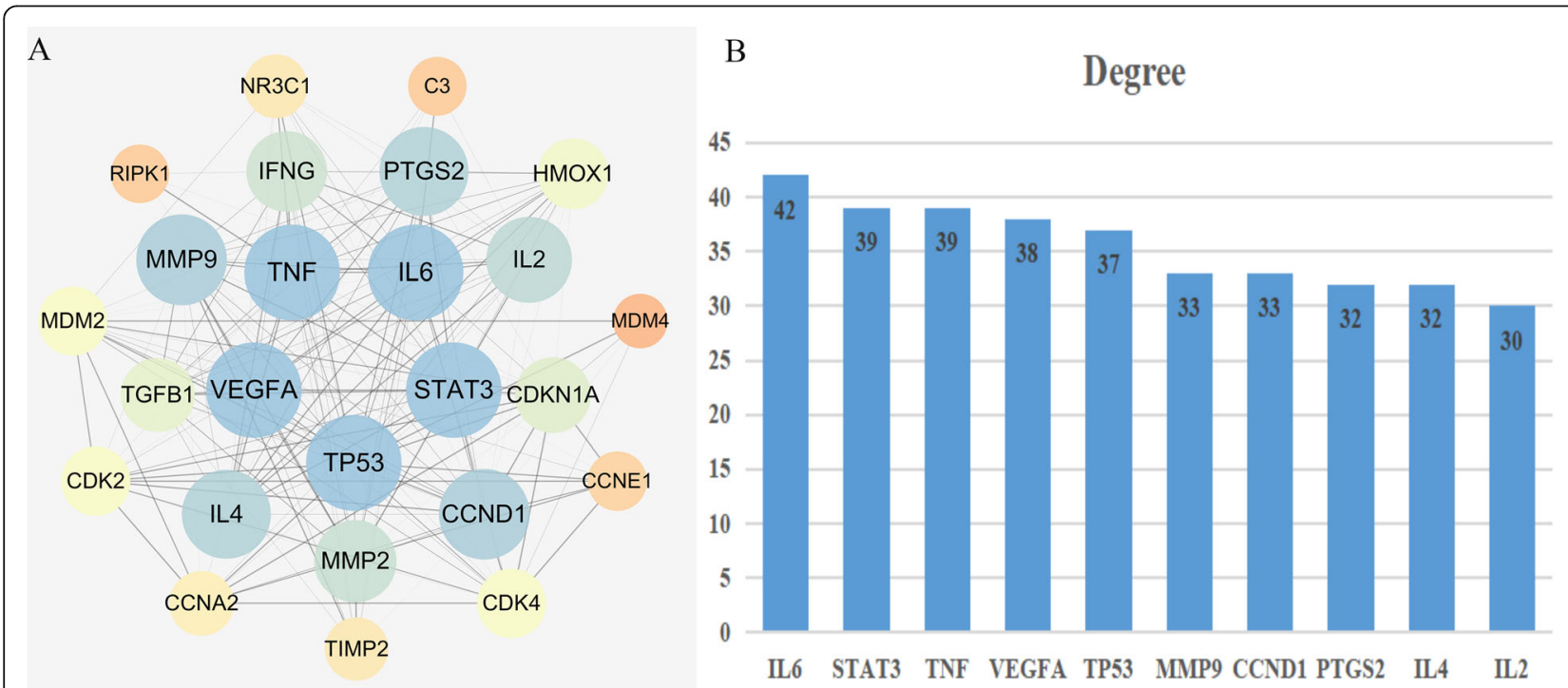

Fig. 5 The PPI networks of RA-SLE-ADR for THH (a) and key targets with the higher degree (b) 
Table 2 Topological parameters for key targets of RA-SLE-ADR (Top 10 by degree)

\begin{tabular}{llll}
\hline Name & Degree & Betweenness Centrality & Closeness Centrality \\
\hline IL6 & 42 & 0.08073 & 0.8889 \\
STAT3 & 39 & 0.05424 & 0.8421 \\
TNF & 39 & 0.05274 & 0.8421 \\
VEGFA & 38 & 0.03849 & 0.8276 \\
TP53 & 37 & 0.03982 & 0.8136 \\
MMP9 & 33 & 0.04720 & 0.7619 \\
CCND1 & 33 & 0.02525 & 0.7619 \\
PTGS2 & 32 & 0.01696 & 0.7500 \\
IL4 & 32 & 0.02743 & 0.7500 \\
IL2 & 30 & 0.02124 & 0.7273 \\
\hline
\end{tabular}

between these promising ingredients of $\mathrm{THH}$ and core targets. Especially, celastrol, L-epigallocatechin, triptonoterpenol and triptophenolide could achieve strong binding activity with MMP9 and STAT3. For example, the docking scores of core targets including MMP9, STAT3, TP53 and triptophenolide were 7.852, 7.869, and 6.152, respectively. Also, triptonoterpenol possessed the stronger binding capacity for MMP9, STAT3 and VEGFA, with the docking scores were $6.545,6.567$ and 6.152, respectively. The detailed of docking results is listed in Table 3. With regards to core targets in Fig. 7, TP53 and VEGFA well interacted with all active principles of THH. For instance, the binding mode of celastrol and TP53 was mainly reflected in two aspects, one was forming hydrogen bonding with Leu 70(A), Val 81(A), and Ala 54(A), the other was hydrophobic interactions with 6 amino acid residues, including Asp 78(A), Cys 76(A), His 53(A), Phe 51(A), Thr 52(A), and Thr 80(A).

\section{Discussion}

$\mathrm{THH}$, an anti-rheumatic herbal that has been widely used in China, is effective for treating autoimmune disease due to its excellent anti-inflammatory and immunosuppressive activities, however, the potential mechanisms of clinical efficacy and safety remain undefined and confused [50]. Therefore, we focused on the benefits and harms of THH during the treatment, at the molecular level, we were interested in the interaction and binding activities between hub targets and active principles of THH in the pathogenesis of dominant diseases and induced ADRs. On the one hand, eight kinds of medicinal chemistry from THH were selected as dominate chemical profiles, including hypodiolide A, hypolide, Lepigallocatechin, tripdiolide, triptolide, triptonide, triptonoterpenol, and celastrol. Moreover, the hub targets corresponding to these active ingredients were determined via combining network topological parameters with PPI network analysis. In total, four targets, namely, STAT3, VEGFA, TP53 and MMP9, might contribute to the pathogenesis of efficacy and safety. On the other hand, unexpectedly, these active ingredients of $\mathrm{THH}$ moderately bind to core targets to exert efficacy and induce toxicity simultaneously. Overall, therapeutic effects and herb-induced disease of $\mathrm{THH}$ were associated with the potential mechanisms that its active compounds played vital role in the regulation of STAT3, VEGFA, TP53 and MMP9. Herein, our study suggested that the combination of network pharmacology prediction and molecular docking simulation might provide some insights into the underlying mechanistic consequences and valuable approaches to characterize molecular mechanism of toxic herbs.

With regard to the active ingredients of $\mathrm{THH}$ which possess efficacy and toxicity concurrently, an anti-

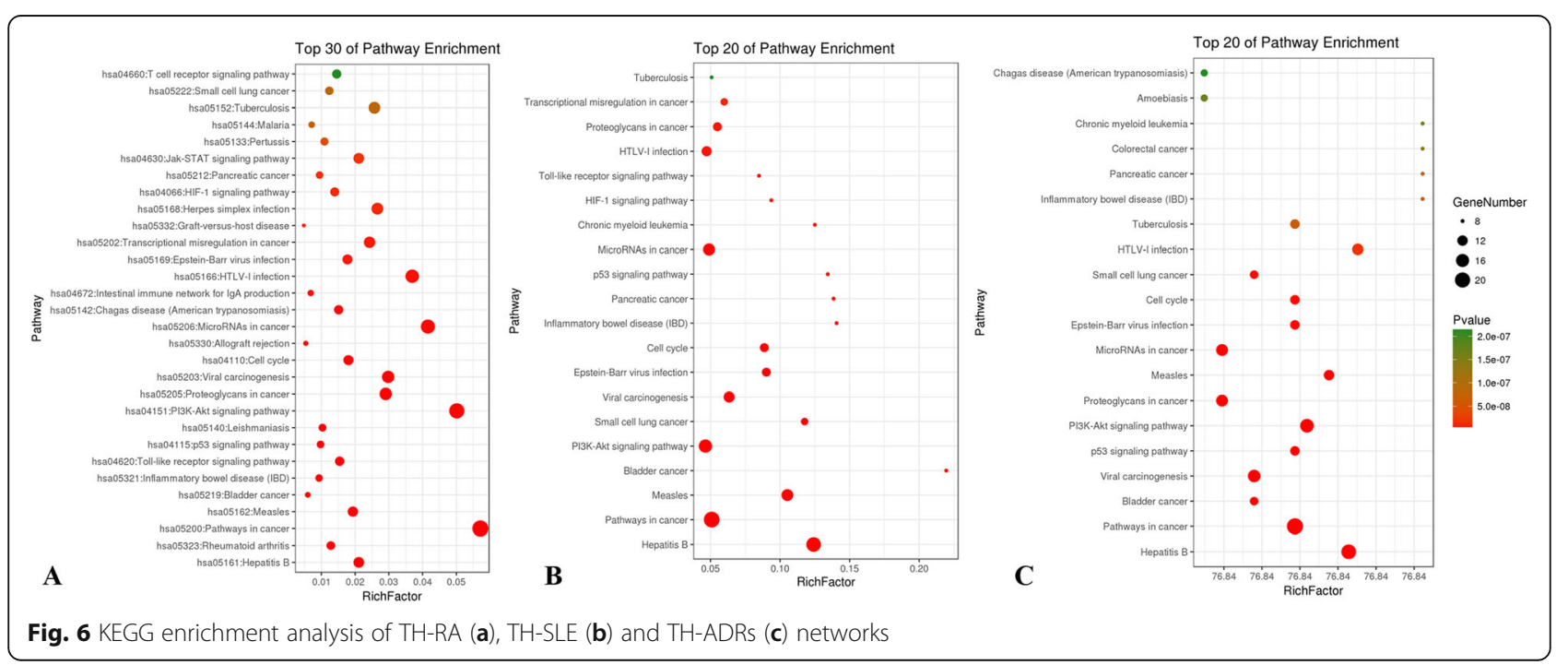




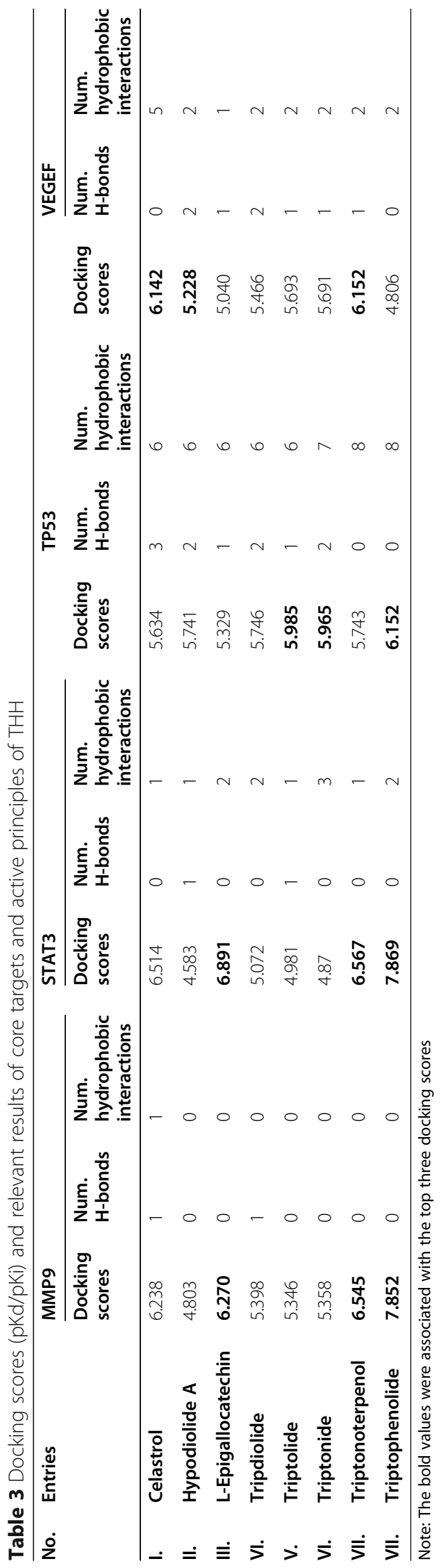




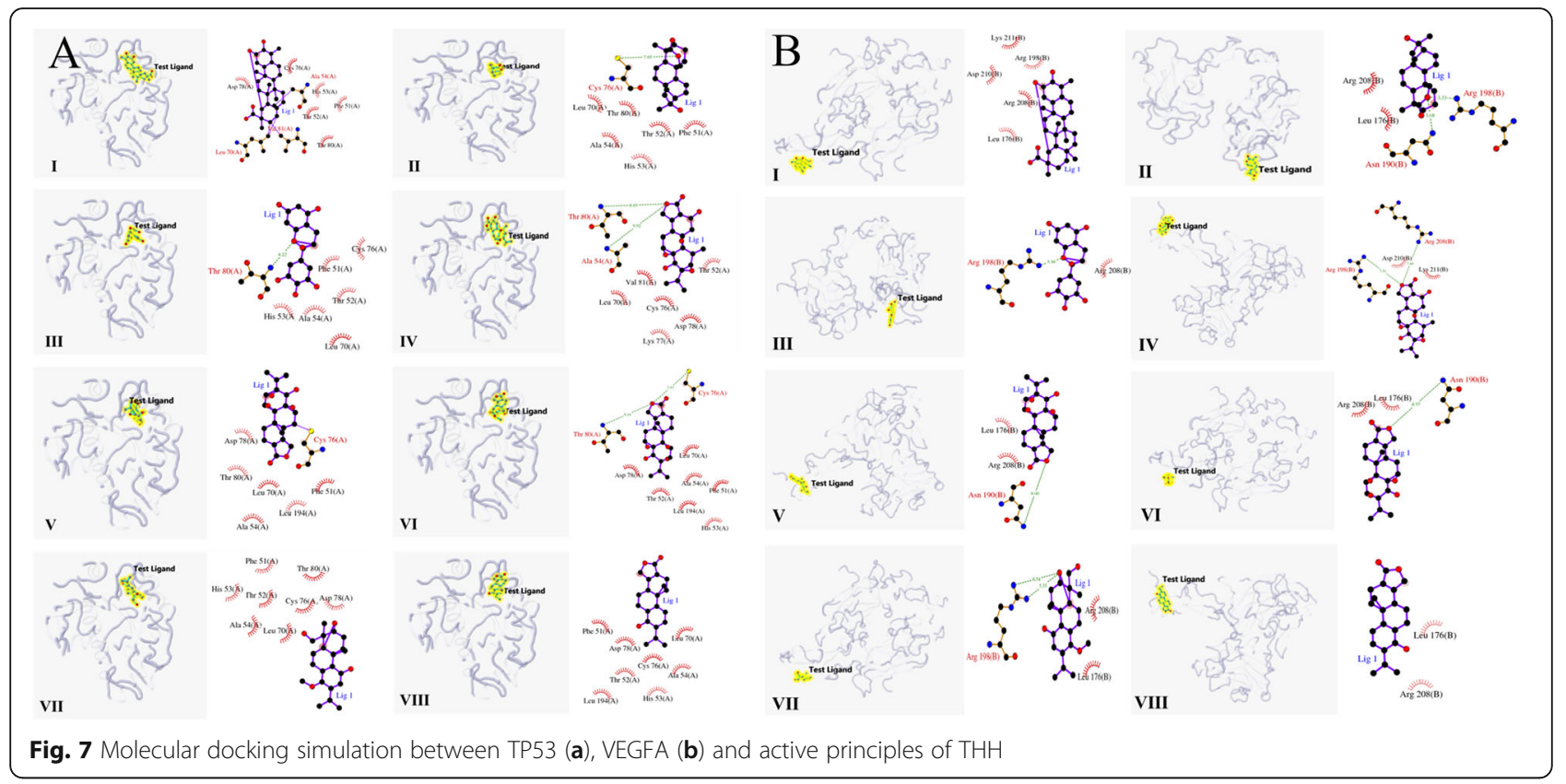

inflammatory and anti-oxidant activities is a bioactive compound, celastrol, is purified from herbs of the Celastraceae family, it can modulate the nuclear factor-kappa B (NF-kB), mitogen-activated protein kinases (MAPK), Janus kinase/ signal transducer and activator of transcription (JAK/STAT), Toll-like receptor (TLR) pathway and so on [51, 52]. In addition, the results of relevant study revealed that except for controlling of autoimmune inflammation, celastrol has the potential of pharmacological treatment of obesity as leptin sensitizer in silico drug screening methods [53, 54]. By contrast, celastrol that involves in inhibition of hERG channel activity and apoptosis could induce cardiotoxicity via the techniques of patch clamp, integrated metabolomics and network toxicology $[55,56]$. Besides, celastrol and triptolide have toxic potencies on hepatocytes that mediated by hepatic CYP450s, affect embryonic development of zebrafish in $\mu \mathrm{M}$ concentrations $[57,58]$. Similarly, triptolide and tripdiolide participate in the production of cytokine and chemokine to significantly ameliorate lupus nephritis in (NZB x NZW) F1 mice, triptolide and triptonide can upregulate the expression of IL-37 via the activation of the ERK1/2 and p38 MAPK pathways to achieve anti-inflammatory functions $[59,60]$.

From the perspective of core targets, strikingly, as a predominant member of extracellular polypeptide ligands like interleukin-(IL)-6-family, STAT3 protein is central in determining the inflammation and immunity, fulfilling fundamental functions in immune cells compared with the other members [61-63]. Additionally, overwhelming evidence suggests that STAT3 has the largest spectrum of potential activators, including various cytokines, hormones, and growth factors. For instance, it has also been implicated in many cellular processes including the transcription or opening of mitochondrial permeability transition pore, cardiomyocyte function, cardiac microenvironment, communicates in cardiomyocytes, cardiac fibroblasts, endothelial cells, smooth muscle cells, inflammatory cells and cardiac neurons $[64,65]$. Other peculiar characteristics of STAT3 cover its ability in inactivation of embryonic lethality, reduction of tubulointerstitial and renal lesions, regulation liver progenitor, development of lung fibrosis [66-69]. Consequently, these functional properties of STAT3 is relevant to unwarranted side effects of THH. Subsequently, VEGFA may be promising markers of RA with clinical significance because it is the most potent proangiogenic molecule promoting the angiogenic phenotype, and it has been proven that its polymorphism is associated with RA susceptibility among Chinese and Mexican patients [70-73]. Also, it has been demonstrated that VEGFA implicated in regulation of cardiovascular development, male fertility, hepatic angiogenesis, glomerular filtration barrier through researches in the field of pharmacology, gene polymorphisms and clinical data [74-79]. TP53 (Cellular tumor antigen p53), a stress response gene, is involved in diverse cell death pathways and its activation, and it always correlates with rheumatoid arthritis, neuronal damage, acute myeloid leukemia, hepatocellular apoptosis [80-84]. As a member of proteases family that is secreted as inactive zymogens, it has been widely accepted that Matrix metalloproteinase 9 (MMP9) is critical in regulating extracellular matrix during the degradation of various extracellular matrix 
proteins, and it is possible pathogenic factor and potential therapeutic target for multi-system disorder [85-87].

Recently, network pharmacology has made a tremendous advance to drug discovery and TCM development [88, 89]. Coincidentally, it is consistent with unique characteristic of TCM or formulas that highlights holistic therapy and synergistic effect via multiple targets and pathways. And this approach has been applied in drug development in consideration of both efficacy and toxicity. Given this, network pharmacology holds the promise of expanding current opportunity space for druggable targets, and deciphering potential mechanism based on the prediction of the target profiles and pharmacological actions of herbal compounds [9092]. Remarkably, molecular docking simulation is an important method in structural molecular biology and computer-assisted drug design [93]. Herein, the combination of these techniques can not only provide powerful and feasible means for investigating the mechanisms for bioactive ingredients, but also reveal the relationships, interconnections and binding free energy between chemicals and corresponding targets [94-96]. According to the results of the molecular docking and protein-ligand interaction profiles in present research, among the four core proteins, TP53 and VEGFA well interacted with all active principles of $\mathrm{THH}$ via more hydrogen bonds and amino acid residues.

\section{Conclusion}

In conclusion, the integrated strategy of network pharmacology and molecular docking simulation was used to illustrate active ingredients and molecular mechanisms of clinical efficacy and safety for THH. Accordingly, therapeutic effects and herb-induced disease of THH were associated with its active compounds, which had the superior binding activities for regulating STAT3, VEGFA, TP53 and MMP9. Taken together, current research provided a comprehensive overview for toxic herbs whose benefits and harms should be balanced during clinical treatment. However, these findings of our results are warranted to validate by further pharmacokinetics and pharmacological researches.

\section{Abbreviations}

ADRs: adverse reactions; BC: betweenness centrality; CC: closeness centrality; CTD: Comparative Toxicogenomics Database; DAVID: The Database for Annotation, Visualization, and Integrated Discovery; DC: degree centrality; GAD: Genetic Association Database; IL6: Interleukin-6 receptor; KEGG: Kyoto Encyclopedia of Genes and Genomes; MMP9: matrix metalloprotein 9; OMIM: Online Mendelian Inheritance in Man; PDB: Protein Data Bank database; PPIs: protein-protein interactions; RA: rheumatoid arthritis; SLE: systemic lupus erythematosus; STAT3: Signal transducers and activators of transcription 3; STRING: Search Tool for the Retrieval of Interacting Genes/ Proteins; TCM: traditional Chinese medicine; TCMSP: Traditional Chinese Medicine Systems Pharmacology Database; THH: Tripterygium hypoglaucum Hutch; TNF: Tumor Necrosis Factor; TP53: Cellular tumor antigen p53;

TTD: Therapeutic Target Database; VEGFA: vascular endothelial growth factor

\section{Acknowledgments}

We would like to appreciate all editors/reviewers for their helpful comments and valuable suggestions.

\section{Authors' contributions}

$D Z, Y D, J$, and BZ: conception and design; DZ, YD, JL, and BZ: Analysis and interpretation of the data; $D Z, Y D, J L, B Z, M Z, Z L$ : Drafting the manuscript DZ, BZ, MZ, ZL: Revising the work critically for important intellectual content; all authors: Study supervision and final approval of the work

\section{Funding}

This work is supported by the National Natural Science Foundation of China (Grant No. 81874349), the Programs Foundation for Leading Talents in State Administration of Traditional Chinese Medicine of China-"Qihuang scholars" Project (10400633210004), and National special support plan for high-level talents (Plan of ten thousand people)-Famous Teacher Program to Professor Bing Zhang. Funding bodies did not involve in the study design and collection, analysis, and interpretation of data.

\section{Availability of data and materials}

Specific study data are available from the authors on request.

Ethics approval and consent to participate

Not applicable.

\section{Consent for publication}

Not applicable.

\section{Competing interests}

The authors declare no competing interests in any aspects.

Received: 1 July 2020 Accepted: 7 January 2021

Published online: 15 January 2021

\section{References}

1. $L v H$, Jiang $L$, Zhu $M$, et al. The genus Tripterygium: a phytochemistry and pharmacological review. Fitoterapia. 2019:137:104190.

2. Li ZY, Wu Q, Yan Z, et al. Prevention of acute GVHD in mice by treatment with Tripterygium hypoglaucum hutch combined with cyclosporin a. Hematology. 2013;18(6):352-9.

3. Brinker $\mathrm{AM}, \mathrm{Ma}$ J, Lipsky PE, et al. Medicinal chemistry and pharmacology of genus Tripterygium (Celastraceae). Phytochemistry. 2007;68(6):732-66.

4. Chen XL, Liu F, Xiao XR, et al. Anti-inflammatory abietanes diterpenoids isolated from Tripterygium hypoglaucum. Phytochemistry. 2018;156:167-75.

5. Lange BM, Fischedick JT, Lange MF, et al. Integrative approaches for the identification and localization of specialized metabolites in Tripterygium roots. Plant Physiol. 2017;173(1):456-69.

6. Tao $X$, Lipsky PE. The Chinese anti-inflammatory and immunosuppressive herbal remedy Tripterygium wilfordii Hook F. Rheum Dis Clin North Am. 2000;26(1):29-viii.

7. Fan D, Parhira S, Zhu GY, et al. Triterpenoids from the stems of Tripterygium regelii. Fitoterapia. 2016;113:69-73.

8. Guo L, Duan L, Liu K, et al. Chemical comparison of Tripterygium wilfordii and Tripterygium hypoglaucum based on quantitative analysis and chemometrics methods. J Pharm Biomed Anal. 2014;95:220-8.

9. Jiang $X$, Huang $X C, A o L$, et al. Total alkaloids of Tripterygium hypoglaucum (levl.) hutch inhibits tumor growth both in vitro and in vivo. J Ethnopharmacol. 2014;151(1):292-8.

10. Gao C, Lou LL, Wang D, et al. Chemical constituents from the roots of Tripterygium wilfordii and their cytotoxic activity. J Asian Nat Prod Res. 2017;19(7):725-31.

11. Yuan $Z$, Hasnat $M$, Liang $P$, et al. The role of inflammasome activation in Triptolide-induced acute liver toxicity. Int Immunopharmacol. 2019;75: 105754.

12. Wang JM, Li JY, Cai H, et al. Nrf2 participates in mechanisms for reducing the toxicity and enhancing the antitumour effect of Radix Tripterygium wilfordii to S180-bearing mice by herbal-processing technology. Pharm Biol. 2019:57(1):437-48

13. Dudics S, Langan D, Meka RR, et al. Natural products for the treatment of autoimmune arthritis: their mechanisms of action, targeted delivery, and interplay with the host microbiome. Int J Mol Sci. 2018;19(9):2508. 
14. Du X, Nyagblordzro M, An L, et al. Pharmacokinetic and toxicological characteristics of Tripterigium glycosides and their derivatives. Curr Drug Metab. 2018;19(7):605-27.

15. Wei $Y$, Wang $D$, Chen $M$, et al. Extrahepatic cytochrome P450s play an insignificant role in triptolide-induced toxicity. Chin Med. 2018;13:23.

16. Liu Q. Triptolide and its expanding multiple pharmacological functions. Int Immunopharmacol. 2011;11(3):377-83.

17. Li XJ, Jiang ZZ, Zhang LY. Triptolide: progress on research in pharmacodynamics and toxicology. J Ethnopharmacol. 2014;155(1):67-79.

18. Wang J, Wang C, Wu J, et al. Oral microemulsion based delivery system for reducing reproductive and kidney toxicity of Tripterygium glycosides. J Microencapsul. 2019;36(6):523-34

19. Yang $Y, M a X L$, Zhang $X H$. Successful pregnancy with tripterygium glycoside-induced premature ovarian insufficiency: a case report. J Int Med Res. 2019:47(5):2274-9.

20. Guo J, Huang Y, Lei $X$, et al. Reproductive systemic toxicity and mechanism of Glucosides of Tripterygium wilfordii hook. F. (GTW). Ann Clin Lab Sci. 2019;49(1):36-49.

21. Zhang C, Sun PP, Guo HT, et al. Safety profiles of Tripterygium wilfordii hook F: a systematic review and meta-analysis. Front Pharmacol. 2016;7:402.

22. Xi Y, Wang W, Wang L, et al. Triptolide induces p53-dependent cardiotoxicity through mitochondrial membrane permeabilization in cardiomyocytes. Toxicol Appl Pharmacol. 2018;355:269-85.

23. Brown AC. Heart toxicity related to herbs and dietary supplements: online table of case reports. Part 4 of 5. J Diet Suppl. 2018;15(4):516-55.

24. Yang $Y$, Wang $W$, Xiong $Z$, et al. Resveratrol protects against triptolideinduced cardiotoxicity through SIRT3 signaling pathway in vivo and in vitro. Pharmazie. 2016;71(9):514-23.

25. Setty AR, Sigal LH. Herbal medications commonly used in the practice of rheumatology: mechanisms of action, efficacy, and side effects. Semin Arthritis Rheum. 2005;34(6):773-84.

26. Chen SR, Dai Y, Zhao J, et al. A mechanistic overview of triptolide and celastrol, natural products from tripterygium wilfordii hook F. Front Pharmacol. 2018;9:104

27. Li L. Renal damage caused by Tripterygium Wilfordii and Tripterygium hypoglaucum. Chinese Journal for Clinicians. 2006:34:9-10 [in Chinese].

28. Hu YB, Chen ZZ, Wen ZJ, et al. Study on the general pharmacology and long-term toxicity of Tripterygium hypoglaucum. Chongqing Journal of Research on Chinese Drugs and Herbs. 2000;41:46-8 [in Chinese].

29. Tang Y, Jiang W, Qiu W, et al. Research of the reproductive toxicity of Kunmingshanhaitang capsule. Study Journal of Traditional Chinese Medicine. 2005;23:1874-6 [in Chinese].

30. Chen CY. TCM database@Taiwan: the world's largest traditional Chinese medicine database for drug screening in silico. PLoS One. 2011;6(1):e15939.

31. Lipinski CA, Lombardo F, Dominy BW, et al. Experimental and computational approaches to estimate solubility and permeability in drug discovery and development settings. Adv Drug Deliv Rev. 2001:46(1-3):3-26.

32. Muegge I. Selection criteria for drug-like compounds. Med Res Rev. 2003; 23(3):302-21.

33. Wang Y, Bryant SH, Cheng T, et al. PubChem BioAssay: 2017 update. Nucleic Acids Res. 2017:45(D1):D955-63.

34. Ru J, Li P, Wang J, et al. TCMSP: a database of systems pharmacology for drug discovery from herbal medicines. J Cheminform. 2014;6:13.

35. Gfeller D, Grosdidier A, Wirth M, et al. SwissTargetPrediction: a web server for target prediction of bioactive small molecules. Nucleic Acids Res. 2014; 42:W32-8

36. Szklarczyk D, Santos A, von Mering C, et al. STITCH 5: augmenting proteinchemical interaction networks with tissue and affinity data. Nucleic Acids Res. 2016;44(D1):D380-4.

37. UniProt CT. UniProt: the universal protein knowledgebase. Nucleic Acids Res. 2018;46(5):2699.

38. UniProt Consortium. UniProt: a hub for protein information. Nucleic Acids Res. 2015;43:D204-12.

39. Chinese Pharmacopoeia Commission. Pharmacopoeia of the People's Republic of China (2015 edition). Beijing: China Medical Science Press. 2015. (in Chinese).

40. Amberger JS, Hamosh A. Searching online Mendelian inheritance in man (OMIM): a knowledgebase of human genes and genetic phenotypes. Curr Protoc Bioinformatics. 2017:58:1.2.1-1.2.12.

41. Li YH, Yu CY, Li XX, et al. Therapeutic target database update 2018: enriched resource for facilitating bench-to-clinic research of targeted therapeutics. Nucleic Acids Res. 2018;46(D1):D1121-7.
42. Becker KG, Barnes KC, Bright TJ, et al. The genetic association database. Nat Genet. 2004;36:431-2.

43. Davis AP, Grondin CJ, Johnson RJ, et al. The comparative Toxicogenomics database: update 2017. Nucleic Acids Res. 2017:45(D1):D972-8.

44. Szklarczyk D, Gable AL, Lyon D, et al. STRING v11: protein-protein association networks with increased coverage, supporting functional discovery in genome-wide experimental datasets. Nucleic Acids Res. 2019; 47(D1):D607-13.

45. Su G, Morris JH, Demchak B, et al. Biological network exploration with Cytoscape 3. Curr Protoc Bioinformatics. 2014;47:8.13.1-24.

46. Huang da W, Sherman BT, Lempicki RA. Systematic and integrative analysis of large gene lists using DAVID bioinformatics resources. Nat Protoc 2009; 4(1):44-57.

47. Kanehisa M, Furumichi M, Tanabe M, et al. KEGG: new perspectives on genomes, pathways, diseases and drugs. Nucleic Acids Res. 2017:45(D1): D353-61.

48. Hsin KY, Matsuoka Y, Asai Y, et al. systemsDock: a web server for network pharmacology-based prediction and analysis. Nucleic Acids Res. 2016; 44(W1):W507-13.

49. Morris GM, Huey R, Lindstrom W, et al. AutoDock4 and AutoDockTools4 automated docking with selective receptor flexibility. J Comput Chem. 2009; 30:2785-91

50. Chakraborty S, Phu M, de Morais TP, et al. The PDB database is a rich source of alpha-helical anti-microbial peptides to combat disease causing pathogens. F1000Res. 2014;3:295.

51. Venkatesha SH, Dudics S, Astry B, Moudgil KD. Control of autoimmune inflammation by celastrol, a natural triterpenoid. Pathog Dis. 2016;74(6): ftw059.

52. Luo D, Guo Y, Cheng Y, et al. Natural product celastrol suppressed macrophage M1 polarization against inflammation in diet-induced obese mice via regulating Nrf2/HO-1, MAP kinase and NF-kB pathways. Aging. 2017:9(10):2069-82

53. Liu J, Lee J, Salazar Hernandez MA, et al. Treatment of obesity with celastrol. Cell. 2015:161(5):999-1011.

54. Pfuhlmann K, Schriever SC, Baumann P, et al. Celastrol-induced weight loss is driven by hypophagia and independent from UCP1. Diabetes. 2018; 67(11):2456-65.

55. Zhao W, Xiao L, Pan L, et al. Cardiac toxicity of Triptergium wilfordii Hook F. may correlate with its inhibition to hERG channel. Heliyon. 2019;5(10): e02527.

56. Liu C, Zhang C, Wang W, et al. Integrated metabolomics and network toxicology to reveal molecular mechanism of celastrol induced cardiotoxicity. Toxicol Appl Pharmacol. 2019;383:114785.

57. Jin C, Wu Z, Wang L, et al. CYP450s-activity relations of Celastrol to interact with Triptolide reveal the reasons of hepatotoxicity of Tripterygium wilfordii. Molecules. 2019;24(11):2162.

58. Wang S, Liu K, Wang $X$, et al. Toxic effects of celastrol on embryonic development of zebrafish (Danio rerio). Drug Chem Toxicol. 2011;34(1):61-5.

59. Tao X, Fan F, Hoffmann V, et al. Effective therapy for nephritis in (NZB X NZW)F1 mice with triptolide and tripdiolide, the principal active components of the Chinese herbal remedy Tripterygium wilfordii hook F. Arthritis Rheum. 2008;58(6):1774-83.

60. He L, Liang Z, Zhao F, et al. Modulation of IL-37 expression by triptolide and triptonide in THP-1 cells. Cell Mol Immunol. 2015;12(4):515-8.

61. Hillmer EJ, Zhang H, Li HS, et al. STAT3 signaling in immunity. Cytokine Growth Factor Rev. 2016;31:1-15.

62. Haddad E. STAT3: too much may be worse than not enough! Blood. 2015; 125(4):583-4.

63. Ying QL, Wray J, Nichols J, et al. The ground state of embryonic stem cell self-renewal. Nature. 2008:453(7194):519-23.

64. Pipicz M, Demján V, Sárközy $M$, et al. Effects of cardiovascular risk factors on cardiac STAT3. Int J Mol Sci. 2018;19(11):3572

65. O'Sullivan KE, Breen EP, Gallagher HC, et al. Understanding STAT3 signaling in cardiac ischemia. Basic Res Cardiol. 2016;111(3):27.

66. Takeda K, Noguchi K, Shi W, et al. Targeted disruption of the mouse Stat3 gene leads to early embryonic lethality. Proc Natl Acad Sci U S A. 1997; 94(8):3801-4.

67. Bienaimé $\mathrm{F}$, Muorah $\mathrm{M}$, Yammine L, et al. Stat3 controls Tubulointerstitial communication during CKD. J Am Soc Nephrol. 2016;27(12):3690-705.

68. Khaliq M, Ko S, Liu Y, et al. Stat3 regulates liver progenitor cell-driven liver regeneration in zebrafish. Gene Expr. 2018;18(3):157-70. 
69. Pedroza M, Le TT, Lewis $K$, et al. STAT-3 contributes to pulmonary fibrosis through epithelial injury and fibroblast-myofibroblast differentiation. FASEB J. 2016;30(1):129-40.

70. Zhang Y, Qiu H, Zhang H, et al. Vascular endothelial growth factor a (VEGF A) polymorphisms in Chinese patients with rheumatoid arthritis. Scand J Rheumatol. 2013:42(5):344-8.

71. Ramírez-Bello J, Cadena-Sandoval D, Fragoso JM, et al. The VEGFA -1154G/a polymorphism is associated with reduced risk of rheumatoid arthritis but not with systemic lupus erythematosus in Mexican women. J Gene Med. 2018;20(6):e3024.

72. Selaas O, Nordal HH, Halse AK, Brun JG, Jonsson R, Brokstad KA. Serum markers in rheumatoid arthritis: a longitudinal study of patients undergoing infliximab treatment. Int J Rheumatol. 2015:2015:276815.

73. Gavrilă Bl, Ciofu C, Stoica V. Biomarkers in rheumatoid arthritis, what is new? J Med Life. 2016;9(2):144-8

74. Zhu D, Fang Y, Gao K, et al. Vegfa impacts early myocardium development in Zebrafish. Int J Mol Sci. 2017;18(2):444.

75. Sargent KM, Clopton DT, Lu N, et al. VEGFA splicing: divergent isoforms regulate spermatogonial stem cell maintenance. Cell Tissue Res. 2016;363(1): $31-45$.

76. Marakhovskaya TA, Butenko EV, Kovalenko KA, et al. Association of growth factors genes with miscarriage. J Reprod Infertil. 2018;19(4):219-28.

77. Langmia IM, Apalasamy YD, Omar SZ, Mohamed Z. Association of VEGFA gene polymorphisms and VEGFA plasma levels with spontaneous preterm birth. Pharmacogenet Genomics. 2015;25(4):199-204.

78. Jin X, Aimaiti $Y$, Chen $Z$, et al. Hepatic stellate cells promote angiogenesis via the TGF- $\beta 1$-Jagged1NEGFA axis. Exp Cell Res. 2018; 373(1-2):34-43.

79. Wang H, Yue Z, Wu J, et al. The accumulation of VEGFA in the glomerular basement membrane and its relationship with podocyte injury and proteinuria in Alport syndrome. PLoS One. 2015;10(8):e0135648.

80. Igarashi $\mathrm{H}$, Hashimoto J, Tomita T, et al. TP53 mutations coincide with the ectopic expression of activation-induced cytidine deaminase in the fibroblast-like synoviocytes derived from a fraction of patients with rheumatoid arthritis. Clin Exp Immunol. 2010;161(1):71-80.

81. $X u X X, B i$ JP, Ping $L$, et al. A network pharmacology approach to determine the synergetic mechanisms of herb couple for treating rheumatic arthritis. Drug Des Devel Ther. 2018;12:967-79.

82. Lu T, Kim P, Luo Y. Tp53 gene mediates distinct dopaminergic neuronal damage in different dopaminergic neurotoxicant models. Neural Regen Res. 2017;12(9):1413-7.

83. Prochazka KT, Pregartner G, Rücker FG, et al. Clinical implications of subclonal TP53 mutations in acute myeloid leukemia. Haematologica. 2019; 104(3):516-23.

84. Verstraelen S, Peers B, Maho W, et al. Phenotypic and biomarker evaluation of zebrafish larvae as an alternative model to predict mammalian hepatotoxicity. J Appl Toxicol. 2016;36(9):1194-206.

85. Atta H, El-Rehany M, Hammam O, et al. Mutant MMP-9 and HGF gene transfer enhance resolution of CCl4-induced liver fibrosis in rats: role of ASH1 and EZH2 methyltransferases repression. PLoS One. 2014;9(11): e112384.

86. Appleby $\mathrm{TC}$, Greenstein AE, Hung $M$, et al. Biochemical characterization and structure determination of a potent, selective antibody inhibitor of human MMP9. J Biol Chem. 2017;292(16):6810-20.

87. Xu L, Cai Z, Yang F, Chen M. Activation-induced upregulation of MMP9 in mast cells is a positive feedback mediator for mast cell activation. Mol Med Rep. 2017;15(4):1759-64.

88. Westerhoff HV. Network-based pharmacology through systems biology. Drug Discov Today Technol. 2015;15:15-6.

89. Wu XM, Wu CF. Network pharmacology: a new approach to unveiling traditional Chinese medicine. Chin J Nat Med. 2015;13(1):1-2.

90. Hopkins AL. Network pharmacology: the next paradigm in drug discovery. Nat Chem Biol. 2008;4(11):682-90.

91. Li S, Zhang B. Traditional Chinese medicine network pharmacology: theory, methodology and application. Chin J Nat Med. 2013;11(2):110-20.

92. Yuan $\mathrm{H}, \mathrm{Ma} \mathrm{Q}$, Cui $\mathrm{H}$, et al. How can synergism of traditional medicines benefit from network pharmacology? Molecules. 2017;22(7):1135.

93. Morris GM, Lim-Wilby M. Molecular docking. Methods Mol Biol. 2008;443: 365-82.

94. Pinzi L, Rastelli G. Molecular docking: shifting paradigms in drug discovery. Int J Mol Sci. 2019;20(18):4331.
95. Zhang X, Wang D, Ren X, et al. System bioinformatic approach through molecular docking, network pharmacology and microarray data analysis to determine the molecular mechanism underlying the effects of rehmanniae Radix Praeparata on cardiovascular diseases. Curr Protein Pept Sci. 2019; 20(10):964-75

96. Pingili RB, Challa SR, Pawar AK, et al. A systematic review on hepatoprotective activity of quercetin against various drugs and toxic agents: evidence from preclinical studies. Phytother Res. 2020;34(1):5-32.

\section{Publisher's Note}

Springer Nature remains neutral with regard to jurisdictional claims in published maps and institutional affiliations.
Ready to submit your research? Choose BMC and benefit from:

- fast, convenient online submission

- thorough peer review by experienced researchers in your field

- rapid publication on acceptance

- support for research data, including large and complex data types

- gold Open Access which fosters wider collaboration and increased citations

- maximum visibility for your research: over $100 \mathrm{M}$ website views per year

At $\mathrm{BMC}$, research is always in progress.

Learn more biomedcentral.com/submissions 